\title{
A Novel Approach to $H_{\infty}$ Control Design for Linear Neutral Time-Delay Systems
}

\author{
Hongwei Xia, ${ }^{1}$ Pingping Zhao, ${ }^{2} \mathrm{Li} \mathrm{Li}^{3}$ Aiguo Wu, ${ }^{2}$ and Guangcheng $\mathrm{Ma}^{1}$ \\ ${ }^{1}$ Space Control and Inertial Technology Research Center, Harbin Institute of Technology, Harbin 150001, China \\ ${ }^{2}$ Shenzhen Graduate School, Harbin Institute of Technology, Shenzhen 518055, China \\ ${ }^{3}$ School of Information Science and Electrical Engineering, Harbin Institute of Technology at Weihai, \\ Weihai 264200, China
}

Correspondence should be addressed to Hongwei Xia; xiahongwei@hit.edu.cn

Received 2 February 2013; Accepted 24 February 2013

Academic Editor: Ligang Wu

Copyright (c) 2013 Hongwei Xia et al. This is an open access article distributed under the Creative Commons Attribution License, which permits unrestricted use, distribution, and reproduction in any medium, provided the original work is properly cited.

This paper is concerned with the problem of $H_{\infty}$ control of linear neutral systems with time-varying delay. Firstly, by applying a novel Lyapunov-Krasovskii functional which is constructed with the idea of delay partitioning approach, appropriate free-weighting matrices, an improved delay-dependent bounded real lemma (BRL) for neutral systems is established. By using the obtained BRL, a delay-dependent sufficient condition for the existence of a state-feedback controller, which ensures asymptotic stability and a prescribed $H_{\infty}$ performance level of the corresponding closed-loop system, is formulated in terms of linear matrix inequalities. Some numerical examples are given to illustrate the effectiveness of the proposed design method.

\section{Introduction}

A neutral time-delay system contains delays both in its states and in its derivatives of states, which occurs in various dynamic systems, such as economical systems, biological systems, metallurgical processing systems, nuclear reactor, power systems, and long-transmission lines in pneumatic and hydraulic systems [1-10]. It has been recognized that time delays can degrade system performance and even result in instability [3-8]. Therefore, researchers have paid considerable attention to the problems of analysis and synthesis for time-delay systems in the last decades (e.g., [1-16]).

In practical applications, however, it is desirable to design a controller such that the closed-loop system is not only stable but also possesses an adequate level of performance $[6,7,10$, 11]. One approach to cope with this problem is the so-called $H_{\infty}$ control approach. The main objective of the $H_{\infty}$ control is to obtain a controller such that the resulted closed system allows a maximum delay size for a fixed $H_{\infty}$ performance bound or achieves a minimum $H_{\infty}$ performance bound for a fixed delay size [10-12]. The conservatism in the $H_{\infty}$ control is hence measured by the allowable delay size or performance bound obtained. Recently, many results on $H_{\infty}$ control of neutral systems have appeared in the literature; see $[4,10-$ $12,16,17]$ and the references therein.

In general, the existing literature for time-delay systems can be roughly divided into two types: delay-independent results [8-10] and delay-dependent ones [16-27]. The former is irrelevant to the delay size while the latter includes the information of delay size. Obviously, it has been recognized that delay-dependent results are generally less conservative than delay-independent ones, particularly when the delay size is time varying $[20-24,28,29]$. In order to further reduce the conservatism, some improved delay-dependent stability conditions are derived by introducing free-weighting matrices in [19]. In fact, Wu et al. and He et al. [20, 28] have proposed some effective methods for dealing with time-delay systems, which employ free-weighting matrices to express the relationships between the terms in the Leibinz-Newton formula. The method therein reduces the conservativeness of methods involving a fixed model transformation. Additional studies can be found in $[8,16,20,25,28,30]$ and references cited therein. 
On the other hand, some other efforts on improving the delay-dependent conditions were made through introducing new Lyapunov-Krasovskii functional. To mention a few, new classes of Lyapunov functional and augmented Lyapunov functional were introduced to study the delay-dependent stability for systems with time-varying delay in [28], which is shown to possess less conservatism than the existing ones. In [21], based on a novel fuzzy Lyapunov-Krasovskii functional which is constructed using a delay partitioning method, a delay-dependent criterion is developed for the stability analysis of fuzzy time-varying state delay systems. In [24], a less conservative delay-dependent robust $H_{\infty}$ control is proposed for uncertain linear systems with a state-delay based on a new Lyapunov-Krasovskii functional. A new criterion of asymptotic stability is derived in [26] by introducing a novel Lyapunov functional with the idea of partitioning the lower bound of the time-varying delay. Recently, there is enormous growth of interest in using the delay partitioning technique to deal with time-delay systems; see, for example [21-23, 26, 3133]. The basic idea of this approach is to evenly partition time delay into several components. By constructing a LyapunovKrasovskii functional (LKF) with every delay component, one can obtain a less conservative stability condition as discussed by F. Gouaisbaut and Peaucelle [22] and Wu et al. $[21,29]$. More results can be found in articles [17, 21-27, 2933 ] and the references therein.

In this context, motivated by Wu et al. [21] and Zhang and Li [23], we will study the delay-dependent $H_{\infty}$ control problem of a class of neutral time-delay systems based on a delay partitioning technique. The remainder of the paper is organized as follows. Section 2 gives problem formulation and a necessary lemma. In Section 3, dividing the delay interval into multiple segments, using the Lyapunov functional technology combined with matrix inequality technology, a new delay-dependent bounded real lemma is proposed. Based on the BRL, a condition for the existence of a state-feedback $H_{\infty}$ controller is introduced in terms of linear matrix inequalities. Numerical examples are given in Section 4, followed by the conclusions, which are presented in Section 5.

Notation. Throughout this paper, " $T$ " stands for matrix transposition. " $I$ " denotes the identity matrix of appropriate dimensions. " $P>0$ " means that $P$ is positive definite. "*" represents the elements below the main diagonal of a symmetric matrix.

\section{Problem Formulations}

Consider a class of linear time-varying discrete neutral system:

$$
\begin{gathered}
\dot{x}(t)-C_{d} \dot{x}(t-\tau)=A x(t)+A_{d} x(t-d(t)) \\
+B w(t)+B_{u} u(t), \quad t>0, \\
z(t)=C x(t)+C_{u} u(t)+D w(t), \\
x(t)=\phi(t), \quad t \in[-r, 0],
\end{gathered}
$$

where $x(t)$ is the state vector; the matrices $A, A_{d}, B, C, C_{d}$, and $D$ are known constant matrices of appropriate dimensions, and the eigenvalue of the matrix $C_{d}, \rho\left(C_{d}\right)$ satisfies $\rho\left(C_{d}\right)<1$. $w(t)$ is the disturbance input that belongs to $L_{2}[0, \infty) . z(t)$ is the controlled output, and $u(t)$ is the controlled input. $\phi(t)(t \in[-r, 0])$ is the system's initial function which is continuous differentiable on $[-r, 0]$. The scalar $\tau$ is a positive constant time delay. Time delay $d(t)$ is a continuously differentiable function, satisfying the following conditions:

$$
\begin{gathered}
0 \leq d(t) \leq h, \\
\dot{d}(t) \leq \mu,
\end{gathered}
$$

where $h$, and $\mu$ are known positive real constants, and it is assumed that $r=\max \{h, \tau\}$. In this paper, we are interested in designing a memoryless state-feedback controller

$$
u(t)=K x(t)
$$

where $K$ is a constant matrix, such that for a given scalar $\gamma$, the following requirements are satisfied:

(I) the corresponding closed-loop system is asymptotically stable when $w(t)=0$;

(II) under zero initial condition (i.e., $x(t)=0(t \in[-r$, $0])$ ), the corresponding closed-loop system satisfies

$$
\|z\|_{2} \leq \gamma\|w\|_{2}, \quad \forall w \in L_{2}[0, \infty),
$$

where $\gamma>0$ is a prescribed scalar.

In obtaining the main results of this paper, the following lemma plays an important role.

Lemma 1 (Schur complement). For the symmetrical matrix $L=\left[\begin{array}{ll}L_{11} & L_{12} \\ L_{12}^{T} & L_{22}\end{array}\right]$, the followings are equivalent:

(1) $L<0$,

(2) $L_{11}<0, \quad L_{22}-L_{12}^{T} L_{11}^{-1} L_{12}<0$,

(3) $L_{22}<0, \quad L_{11}-L_{12} L_{22}^{-1} L_{12}^{T}<0$.

\section{Main Results}

In this section, we discuss the problem of $H_{\infty}$ performance and state-feedback $H_{\infty}$ controller design of system (1).

3.1. $H_{\infty}$ Performance Analysis. In the following theorem, we present a new version of delay-dependent bounded real lemma for neutral system (1) with $u(t) \equiv 0$; that is, we consider the following system:

$$
\begin{gathered}
\dot{x}(t)-C_{d} \dot{x}(t-\tau)=A x(t)+A_{d} x(t-d(t))+B w(t), \\
z(t)=C x(t)+D w(t) .
\end{gathered}
$$

Theorem 2. For given positive scalars $h>0$ and $\mu>0$, the neutral system (6) with delay restrictions (2) is asymptotically stable and satisfies $\|z\|_{2} \leq \gamma\|w\|_{2}$ for any nonzero $w(t) \epsilon$ $L_{2}[0, \infty)$ under the zero initial condition if there exist matrices 
$P=P^{T}>0, Q_{i}=Q_{i}^{T} \geq 0(i=1, \ldots, 4), R=R^{T}>0, Z=$ $Z^{T}>0$,

$$
X=\left[\begin{array}{cccccc}
X_{11} & X_{12} & X_{13} & X_{14} & X_{15} & X_{16} \\
* & X_{22} & X_{23} & X_{24} & X_{25} & X_{26} \\
* & * & X_{33} & X_{34} & X_{35} & X_{36} \\
* & * & * & X_{44} & X_{45} & X_{46} \\
* & * & * & * & X_{55} & X_{56} \\
* & * & * & * & * & X_{66}
\end{array}\right] \geq 0
$$

and free-weighting matrices $N_{i}(i=1, \ldots, 6)$ with appropriate dimensions, such that the following LMIs hold:

$$
\Phi=\left[\begin{array}{ccccccccc}
\Phi_{11} & \Phi_{12} & \Phi_{13} & \Phi_{14} & \Phi_{15} & \Phi_{16} & A^{T} H & 0 & C^{T} \\
* & \Phi_{22} & \Phi_{23} & \Phi_{24} & \Phi_{25} & \Phi_{26} & 0 & 0 & 0 \\
* & * & \Phi_{33} & \Phi_{34} & \Phi_{35} & \Phi_{36} & A_{d}^{T} H & 0 & 0 \\
* & * & * & \Phi_{44} & \Phi_{45} & \Phi_{46} & 0 & 0 & 0 \\
* & * & * & * & \Phi_{55} & \Phi_{56} & 0 & 0 & 0 \\
* & * & * & * & * & \Phi_{66} & C_{d}^{T} H & 0 & 0 \\
* & * & * & * & * & * & -H & 0 & 0 \\
* & * & * & * & * & * & * & -\gamma^{2} I & D^{T} \\
* & * & * & * & * & * & * & * & -I
\end{array}\right]
$$

$<0$,

$$
\Psi=\left[\begin{array}{ccccccc}
X_{11} & X_{12} & X_{13} & X_{14} & X_{15} & X_{16} & N_{1} \\
* & X_{22} & X_{23} & X_{24} & X_{25} & X_{26} & N_{2} \\
* & * & X_{33} & X_{34} & X_{35} & X_{36} & N_{3} \\
* & * & * & X_{44} & X_{45} & X_{46} & N_{4} \\
* & * & * & * & X_{55} & X_{56} & N_{5} \\
* & * & * & * & * & X_{66} & N_{6} \\
* & * & * & * & * & * & Z
\end{array}\right] \geq 0,
$$

where

$$
\begin{gathered}
\Phi_{11}=P A+A^{T} P+Q_{1}+N_{1}+N_{1}^{T}+h X_{11}, \\
\Phi_{12}=N_{2}^{T}+h X_{12}, \quad \Phi_{13}=P A_{d}-N_{1}+N_{3}^{T}+h X_{13}, \\
\Phi_{14}=N_{4}^{T}+h X_{14}, \quad \Phi_{15}=N_{5}^{T}+h X_{15}, \\
\Phi_{16}=P C_{d}+N_{6}^{T}+h X_{16}, \\
\Phi_{22}=-\left(1-\frac{\mu}{2}\right) Q_{1}+Q_{2}+h X_{22}, \\
\Phi_{23}=-N_{2}+h X_{23}, \quad \Phi_{24}=h X_{24}, \\
\Phi_{25}=h X_{25}, \quad \Phi_{26}=h X_{26}, \\
\Phi_{33}=-(1-\mu) Q_{2}+Q_{3}-N_{3}-N_{3}^{T}+h X_{33}, \\
\Phi_{34}=-N_{4}^{T}+h X_{34}, \quad \Phi_{35}=-N_{5}^{T}+h X_{35} \\
\Phi_{36}=-N_{6}^{T}+h X_{36}, \\
\Phi_{44}=-\left(1-\frac{\mu}{2}\right) Q_{3}+Q_{4}+h X_{44},
\end{gathered}
$$

$$
\begin{aligned}
& \Phi_{45}=h X_{45}, \quad \Phi_{46}=h X_{46}, \\
& \Phi_{55}=-Q_{4}+h X_{55}, \quad \Phi_{56}=h X_{56} \text {, } \\
& \Phi_{66}=-R+h X_{66}, \quad H=R+h Z
\end{aligned}
$$

Proof. Under the condition of the theorem, we first show the asymptotic stability of system (6). To this end, we consider system (6) with $w(t)=0$, that is,

$$
\dot{x}(t)-C_{d} \dot{x}(t-\tau)=A x(t)+A_{d} x(t-d(t)) .
$$

Inspired by the works of [23], we divided the delay interval $[0, h]$ into $[0, d(t) / 2],[d(t) / 2, d(t)],[d(t),(d(t)+h) / 2]$, and $[(d(t)+h) / 2, h]$ for system (6). Corresponding to such a division, the following Lyapunov-Krasovskii functional is chosen for this system:

$$
\begin{aligned}
V\left(t, x_{t}\right)= & x^{T}(t) P x(t)+\int_{t-d(t) / 2}^{t} x^{T}(s) Q_{1} x(s) d s \\
& +\int_{t-d(t)}^{t-d(t) / 2} x^{T}(s) Q_{2} x(s) d s \\
& +\int_{t-(d(t)+h) / 2}^{t-d(t)} x^{T}(s) Q_{3} x(s) d s \\
& +\int_{t-h}^{t-(d(t)+h) / 2} x^{T}(s) Q_{4} x(s) d s \\
& +\int_{t-\tau}^{t} \dot{x}^{T}(s) R \dot{x}(s) d s \\
& +\int_{-h}^{0} \int_{t+\theta}^{t} \dot{x}^{T}(s) Z \dot{x}(s) d s d \theta,
\end{aligned}
$$

where $P=P^{T}>0, Q_{i}=Q_{i}^{T} \geq 0(i=1, \ldots, 4), R=R^{T}>0$, and $Z=Z^{T}>0$ are matrices to be determined. By using the Leibniz-Newton formula, one has

$$
x(t-d(t))=x(t)-\int_{t-d(t)}^{t} \dot{x}(s) d s .
$$

Since (13) can be rewritten as $x(t)-x(t-d(t))-\int_{t-d(t)}^{t} \dot{x}(s) d s=$ 0 . Due to this relation, one can introduce some appropriate dimensional matrices $N_{i}(i=1, \ldots, 6)$, such that

$$
\begin{aligned}
& 2\left[x^{T}(t) N_{1}+x^{T}\left(t-\frac{d(t)}{2}\right) N_{2}+x^{T}(t-d(t)) N_{3}\right. \\
& \quad+x^{T}\left(t-\frac{d(t)+h}{2}\right) N_{4}+x^{T}(t-h) N_{5} \\
& \left.\quad+\dot{x}^{T}(t-\tau) N_{6}\right] \\
& \times\left[x(t)-x(t-d(t))-\int_{t-d(t)}^{t} \dot{x}(s) d s\right]=0 .
\end{aligned}
$$

Moreover, it follows from (2) that for any appropriate dimensional matrix $X \geq 0$,

$$
h \eta^{T}(t) X \eta(t)-\int_{t-d(t)}^{t} \eta^{T}(t) X \eta(t) d s \geq 0,
$$


where

$$
\eta(t)=\left[x^{T}(t) x^{T}\left(t-\frac{d(t)}{2}\right) x^{T}(t-d(t)) x^{T}\left(t-\frac{d(t)+h}{2}\right) x^{T}(t-h) \dot{x}^{T}(t-\tau)\right]^{T} .
$$

According to (14) and (15), and taking the time derivative of the Lyapunov-Krasovskii functional candidate (12) gives that

$$
\begin{aligned}
& \dot{V}\left(t, x_{t}\right) \\
& =2 x^{T}(t) P \dot{x}(t)+x^{T}(t) Q_{1} x(t) \\
& -\left(1-\frac{\dot{d}(t)}{2}\right) x^{T}\left(t-\frac{d(t)}{2}\right) Q_{1} x\left(t-\frac{d(t)}{2}\right) \\
& +\left(1-\frac{\dot{d}(t)}{2}\right) x^{T}\left(t-\frac{d(t)}{2}\right) Q_{2} x\left(t-\frac{d(t)}{2}\right) \\
& -(1-\dot{d}(t)) x^{T}(t-d(t)) Q_{2} x(t-d(t)) \\
& +\left(1-\frac{\dot{d}(t)}{2}\right) x^{T}\left(t-\frac{d(t)}{2}\right) Q_{2} x\left(t-\frac{d(t)}{2}\right) \\
& -(1-\dot{d}(t)) x^{T}(t-d(t)) Q_{2} x(t-d(t)) \\
& +(1-\dot{d}(t)) x^{T}(t-d(t)) Q_{3} x(t-d(t)) \\
& -\left(1-\frac{\dot{d}(t)}{2}\right) x^{T}\left(t-\frac{d(t)+h}{2}\right) Q_{3} \\
& \times x\left(t-\frac{d(t)+h}{2}\right) \\
& +\left(1-\frac{\dot{d}(t)}{2}\right) x^{T}\left(t-\frac{d(t)+h}{2}\right) Q_{4} \\
& \times x\left(t-\frac{d(t)+h}{2}\right)-x^{T}(t-h) Q_{4} x(t-h) \\
& +\dot{x}^{T}(t) R \dot{x}(t)-\dot{x}^{T}(t-\tau) R \dot{x}(t-\tau) \\
& +h \dot{x}^{T}(t) Z \dot{x}(t)-\int_{t-h}^{t} \dot{x}^{T}(s) Z \dot{x}(s) d s \\
& \leq 2 x^{T}(t) P\left[A x(t)+A_{d} x(t-d(t))+C_{d} \dot{x}(t-\tau)\right] \\
& +x^{T}(t) Q_{1} x(t) \\
& -\left(1-\frac{\mu}{2}\right) x^{T}\left(t-\frac{d(t)}{2}\right) Q_{1} x\left(t-\frac{d(t)}{2}\right) \\
& +\left(1-\frac{\dot{d}(t)}{2}\right) x^{T}\left(t-\frac{d(t)}{2}\right) Q_{2} x\left(t-\frac{d(t)}{2}\right) \\
& -(1-\mu) x^{T}(t-d(t)) Q_{2} x(t-d(t)) \\
& +(1-\dot{d}(t)) x^{T}(t-d(t)) Q_{3} x(t-d(t))
\end{aligned}
$$

$$
\begin{aligned}
& -\left(1-\frac{\mu}{2}\right) x^{T}\left(t-\frac{d(t)+h}{2}\right) Q_{3} x\left(t-\frac{d(t)+h}{2}\right) \\
& +\left(1-\frac{\dot{d}(t)}{2}\right) x^{T}\left(t-\frac{d(t)+h}{2}\right) Q_{4} \\
& \times x\left(t-\frac{d(t)+h}{2}\right)-x^{T}(t-h) Q_{4} x(t-h) \\
& +\dot{x}^{T}(t) R \dot{x}(t)-\dot{x}^{T}(t-\tau) R \dot{x}(t-\tau) \\
& +h \dot{x}^{T}(t) Z \dot{x}(t)-\int_{t-d(t)}^{t} \dot{x}^{T}(s) Z \dot{x}(s) d s \\
& +2\left[x^{T}(t) N_{1}+x^{T}\left(t-\frac{d(t)}{2}\right) N_{2}\right. \\
& +x^{T}(t-d(t)) N_{3}+x^{T}\left(t-\frac{d(t)+h}{2}\right) N_{4} \\
& \left.+x^{T}(t-h) N_{5}+\dot{x}^{T}(t-\tau) N_{6}\right] \\
& \times\left[x(t)-x(t-d(t))-\int_{t-d(t)}^{t} \dot{x}(s) d s\right] \\
& +h \eta^{T}(t) X \eta(t)-\int_{t-d(t)}^{t} \eta^{T}(t) X \eta(t) d s \\
& =\eta^{T}(t) \Xi \eta(t)-\int_{t-d(t)}^{t} \xi^{T}(t, s) \Psi \xi(t, s) d s \\
& -\frac{\dot{d}(t)}{2} x^{T}\left(t-\frac{d(t)}{2}\right) Q_{2} x\left(t-\frac{d(t)}{2}\right) \\
& -\dot{d}(t) x^{T}(t-d(t)) Q_{3} x(t-d(t)) \\
& -\frac{\dot{d}(t)}{2} x^{T}\left(t-\frac{d(t)+h}{2}\right) Q_{4} x\left(t-\frac{d(t)+h}{2}\right),
\end{aligned}
$$

where

$$
\begin{gathered}
\xi(t)=\left[\begin{array}{lllll}
\eta^{T}(t) & \dot{x}^{T}(s)
\end{array}\right]^{T}, \\
\Xi=\left[\begin{array}{cccccc}
\Phi_{11}+A^{T} H A & \Phi_{12} & \Phi_{13}+A^{T} H A_{d} & \Phi_{14} & \Phi_{15} & \Phi_{16}+A^{T} H C_{d} \\
* & \Phi_{22} & \Phi_{23} & \Phi_{24} & \Phi_{25} & \Phi_{26} \\
* & * & \Phi_{33}+A_{d}^{T} H A_{d} & \Phi_{34} & \Phi_{35} & \Phi_{36}+A_{d}^{T} H C_{d} \\
* & * & * & \Phi_{44} & \Phi_{45} & \Phi_{46} \\
* & * & * & * & \Phi_{55} & \Phi_{56} \\
* & * & * & * & * & \Phi_{66}+C_{d}^{T} H C_{d}
\end{array}\right]
\end{gathered}
$$

and $\Psi$ is denoted in (9). The last three items of (17) are not more than zero since $Q_{i} \geq 0(i=2, \ldots, 4)$. Therefore, if 
$\Xi<0$ and $\Psi \geq 0$, there exists a positive scalar $\varepsilon$ such that $\dot{V}\left(t, x_{t}\right) \leq-\varepsilon\|x(t)\|^{2}$, which guarantees system (6) is asymptotically stable. By Lemma 1 , we can conclude that if the matrix inequality (8) is feasible then the inequality $\Xi<0$ is feasible. This implies that system (6) is asymptotically stable if LMIs (8) and (9) are feasible.

Next, we shall establish the $H_{\infty}$ performance of system (6) under zero initial condition. To this end, we introduce

$$
J_{\gamma}=\int_{0}^{t}\left(z^{T}(\theta) z(\theta)-\gamma^{2} w^{T}(\theta) w(\theta)\right) d \theta
$$

where $t>0$.

By combining (17) with those results analyzed above, now it is interesting to note that

$$
\dot{V}\left(t, x_{t}\right) \leq \eta^{T}(t) \Xi \eta(t)-\int_{t-d(t)}^{t} \xi^{T}(t, s) \Psi \xi(t, s) d s
$$

Considering zero initial condition, it is easy to see that for any nonzero $w \in L_{2}[0, \infty)$ and $t>0$, the following expression holds.

$$
\begin{aligned}
J_{\gamma}= & \int_{0}^{t}\left(z^{T}(\theta) z(\theta)-\gamma^{2} w^{T}(\theta) w(\theta)+\dot{V}(\theta)\right) d \theta-V(t) \\
\leq & \int_{0}^{t}\left(z^{T}(\theta) z(\theta)-\gamma^{2} w^{T}(\theta) w(\theta)+\dot{V}(\theta)\right) d \theta \\
= & \int_{0}^{t} \bar{\eta}^{T}(\theta) \Xi^{\prime} \bar{\eta}(\theta) d \theta \\
& -\int_{0}^{t} \int_{\theta-d(\theta)}^{\theta} \xi^{T}(\theta, s) \Psi \xi(\theta, s) d s d \theta,
\end{aligned}
$$

where

$$
\Xi^{\prime}=\left[\begin{array}{ccccccc}
\Phi_{11}+A^{T} H A+C^{T} C & \Phi_{12} & \Phi_{13}+A^{T} H A_{d} & \Phi_{14} & \Phi_{15} & \Phi_{16}+A^{T} H C_{d} & C^{T} D \\
* & \Phi_{22} & \Phi_{23} & \Phi_{24} & \Phi_{25} & \Phi_{26} & 0 \\
* & * & \Phi_{33}+A_{d}^{T} H A_{d} & \Phi_{34} & \Phi_{35} & \Phi_{36}+A_{d}^{T} H C_{d} & 0 \\
* & * & * & \Phi_{44} & \Phi_{45} & \Phi_{46} & 0 \\
* & * & * & * & \Phi_{55} & \Phi_{56} & 0 \\
* & * & * & * & * & \Phi_{66}+C_{d}^{T} H C_{d} & 0 \\
* & * & * & * & * & * & D^{T} D-\gamma^{2} I
\end{array}\right]<0 .
$$

By carrying out some algebraic manipulations, the aforementioned matrix inequality (22) can be rewritten as follows:

$$
\left[\begin{array}{cccccccc}
\Phi_{11} & \Phi_{12} & \Phi_{13} & \Phi_{14} & \Phi_{15} & \Phi_{16} & A^{T} H & 0 \\
* & \Phi_{22} & \Phi_{23} & \Phi_{24} & \Phi_{25} & \Phi_{26} & 0 & 0 \\
* & * & \Phi_{33} & \Phi_{34} & \Phi_{35} & \Phi_{36} & A_{d}^{T} H & 0 \\
* & * & * & \Phi_{44} & \Phi_{45} & \Phi_{46} & 0 & 0 \\
* & * & * & * & \Phi_{55} & \Phi_{56} & 0 & 0 \\
* & * & * & * & * & \Phi_{66} & C_{d}^{T} H & 0 \\
* & * & * & * & * & * & -H & 0 \\
* & * & * & * & * & * & * & -\gamma^{2} I
\end{array}\right]
$$

$$
+\left[\begin{array}{c}
C^{T} \\
0 \\
0 \\
0 \\
0 \\
0 \\
0 \\
D^{T}
\end{array}\right] \times\left[\begin{array}{llllllll}
C & 0 & 0 & 0 & 0 & 0 & 0 & D
\end{array}\right]<0 .
$$

By Schur complement (Lemma 1) and some matrices primary manipulations, it is easy to see that the abovementioned matrix inequality (23) is equivalent to (8).
Combining (8) and (9), we have $\bar{\eta}^{T}(t) \Xi^{\prime} \bar{\eta}(t) \leq 0$ for any $\bar{\eta}(t)$. Therefore, the following expression holds for any $t>0$ :

$$
J_{\gamma}=\int_{0}^{t}\left(z^{T}(\theta) z(\theta)-\gamma^{2} w^{T}(\theta) w(\theta)\right) d \theta \leq 0 .
$$

By letting $t \rightarrow \infty$, we lead to

$$
\int_{0}^{\infty} z^{T}(\theta) z(\theta) d \theta \leq \gamma^{2} \int_{0}^{\infty} w^{T}(\theta) w(\theta) d \theta .
$$

And hence, (4) is satisfied for any nonzero $w(t) \in L_{2}[0, \infty)$. The proof is thus completed.

Remark 3. Theorem 2 presents an improved bounded real lemma for linear neutral system with time-varying delay by defining a novel Lyapunov-Krasovskii functional. The merit of the proposed BRL lies in its reduced conservatism, which is based on a time-delay fractioning approach.

Remark 4. About the delay partitioning technique, the classical approach is to represent the time delay as two parts: constant part and time-varying part, and then a LyapunovKrasovskii functional is introduced by applying the idea of delay partitioning to the constant part. However, in this brief, we partition the whole time-varying delay interval into multiparts and a novel Lyapunov-Krasovskii functional (LKF) is constructed with every delay component. Then, we 
obtain a time-varying LKF since it is dependent on the timevarying delay, which constitutes the major difference from most existing results in the literature.

3.2. $H_{\infty}$ Control of Neutral Time-Delay Systems. Now, we are in a position to state the $H_{\infty}$ control result based on the BRL derived in the previous section. A sufficient condition under which there exists a memoryless state-feedback $H_{\infty}$ controller for the neutral system (1) is given in Theorem 5.

Theorem 5. For given positive scalars $\mu$ and $\lambda$, there exists a state-feedback controller in the form of (3) such that the resulting closed-loop system satisfies the requirements (I) and
(II) if there exist matrices $\widetilde{P}>0, \widetilde{Q}_{i}>0(i=1, \ldots, 4), \widetilde{R}>0$, $\widetilde{Z}>0$,

$$
\widetilde{X}=\left[\begin{array}{cccccc}
\widetilde{X}_{11} & \widetilde{X}_{12} & \widetilde{X}_{13} & \widetilde{X}_{14} & \widetilde{X}_{15} & \widetilde{X}_{16} \\
* & \widetilde{X}_{22} & \widetilde{X}_{23} & \widetilde{X}_{24} & \widetilde{X}_{25} & \widetilde{X}_{26} \\
* & * & \widetilde{X}_{33} & \widetilde{X}_{34} & \widetilde{X}_{35} & \widetilde{X}_{36} \\
* & * & * & \widetilde{X}_{44} & \widetilde{X}_{45} & \widetilde{X}_{46} \\
* & * & * & * & \widetilde{X}_{55} & \widetilde{X}_{56} \\
* & * & * & * & * & \widetilde{X}_{66}
\end{array}\right] \geq 0
$$

free-weighting matrices $\widetilde{N}_{i}(i=1, \ldots, 6)$ of appropriate dimensions, and a scalar $\gamma>0$, such that the following matrix inequalities hold:

$$
\begin{aligned}
& {\left[\begin{array}{ccccccccc}
\widetilde{\Phi}_{11} & \widetilde{\Phi}_{12} & \widetilde{\Phi}_{13} & \widetilde{\Phi}_{14} & \widetilde{\Phi}_{15} & \widetilde{\Phi}_{16} & \lambda \widetilde{P} B^{T}+\lambda Y^{T} B_{u}^{T} & 0 & \widetilde{P} C^{T}+Y^{T} C_{u}^{T} \\
* & \widetilde{\Phi}_{22} & \widetilde{\Phi}_{23} & \widetilde{\Phi}_{24} & \widetilde{\Phi}_{25} & \widetilde{\Phi}_{26} & 0 & 0 & 0 \\
* & * & \widetilde{\Phi}_{33} & \widetilde{\Phi}_{34} & \widetilde{\Phi}_{35} & \widetilde{\Phi}_{36} & \lambda \widetilde{P} A_{d}^{T} & 0 & 0 \\
* & * & * & \widetilde{\Phi}_{44} & \widetilde{\Phi}_{45} & \widetilde{\Phi}_{46} & 0 & 0 & 0 \\
* & * & * & * & \widetilde{\Phi}_{55} & \widetilde{\Phi}_{56} & 0 & 0 & 0 \\
* & * & * & * & * & \widetilde{\Phi}_{66} & \lambda \widetilde{P} C_{d}^{T} & 0 & 0 \\
* & * & * & * & * & * & -\lambda \widetilde{P} & 0 & 0 \\
* & * & * & * & * & * & * & -\gamma^{2} I & D^{T} \\
* & * & * & * & * & * & * & * & -I
\end{array}\right]<0} \\
& \widetilde{\Psi}=\left[\begin{array}{ccccccc}
\widetilde{X}_{11} & \widetilde{X}_{12} & \widetilde{X}_{13} & \widetilde{X}_{14} & \widetilde{X}_{15} & \widetilde{X}_{16} & \widetilde{N}_{1} \\
* & \widetilde{X}_{22} & \widetilde{X}_{23} & \widetilde{X}_{24} & \widetilde{X}_{25} & \widetilde{X}_{26} & \widetilde{N}_{2} \\
* & * & \widetilde{X}_{33} & \widetilde{X}_{34} & \widetilde{X}_{35} & \widetilde{X}_{36} & \widetilde{N}_{3} \\
* & * & * & \widetilde{X}_{44} & \widetilde{X}_{45} & \widetilde{X}_{46} & \widetilde{N}_{4} \\
* & * & * & * & \widetilde{X}_{55} & \widetilde{X}_{56} & \widetilde{N}_{5} \\
* & * & * & * & * & \widetilde{X}_{66} & \widetilde{N}_{6} \\
* & * & * & * & * & * & \widetilde{Z}
\end{array}\right] \geq 0,
\end{aligned}
$$

where

$$
\begin{gathered}
\widetilde{\Phi}_{11}=B_{u} Y+Y^{T} B_{u}^{T}+\widetilde{P}^{T}+B \widetilde{P}+\widetilde{Q}_{1}+\widetilde{N}_{1}+\widetilde{N}_{1}^{T}+h \widetilde{X}_{11}, \\
\widetilde{\Phi}_{12}=\widetilde{N}_{2}^{T}+h \widetilde{X}_{12}, \\
\widetilde{\Phi}_{13}=A_{d} \widetilde{P}-\widetilde{N}_{1}+\widetilde{N}_{3}^{T}+h \widetilde{X}_{13}, \\
\widetilde{\Phi}_{14}=\widetilde{N}_{4}^{T}+h \widetilde{X}_{14}, \quad \widetilde{\Phi}_{15}=\widetilde{N}_{5}^{T}+h \widetilde{X}_{15} \\
\widetilde{\Phi}_{16}=C_{d} \widetilde{P}^{T} \widetilde{N}_{6}^{T}+h \widetilde{X}_{16}, \\
\widetilde{\Phi}_{22}=-\left(1-\frac{\mu}{2}\right) \widetilde{Q}_{1}+\widetilde{Q}_{2}+h \widetilde{X}_{22}, \\
\widetilde{\Phi}_{24}=h \widetilde{X}_{24}, \quad \widetilde{\Phi}_{23}=-\widetilde{N}_{2}+h \widetilde{X}_{23},
\end{gathered}
$$

$$
\begin{gathered}
\widetilde{\Phi}_{33}=-(1-\mu) \widetilde{Q}_{2}+\widetilde{Q}_{3}-\widetilde{N}_{3}-\widetilde{N}_{3}^{T}+h \widetilde{X}_{33}, \\
\widetilde{\Phi}_{34}=-\widetilde{N}_{4}+h \widetilde{X}_{34}, \quad \widetilde{\Phi}_{35}=-\widetilde{N}_{5}+h \widetilde{X}_{35}, \\
\widetilde{\Phi}_{36}=-\widetilde{N}_{6}+h \widetilde{X}_{36}, \\
\widetilde{\Phi}_{44}=\left(\frac{\mu}{2}-1\right) \widetilde{Q}_{3}+\widetilde{Q}_{4}+h \widetilde{X}_{44}, \\
\widetilde{\Phi}_{45}=h \widetilde{X}_{45}, \quad \widetilde{\Phi}_{46}=h \widetilde{X}_{46}, \\
\widetilde{\Phi}_{55}=-\widetilde{Q}_{4}+h \widetilde{X}_{55}, \quad \widetilde{\Phi}_{56}=h \widetilde{X}_{56} \\
\widetilde{\Phi}_{66}=-\widetilde{R}+h \widetilde{X}_{66} .
\end{gathered}
$$

In this case, an $H_{\infty}$ state-feedback controller can be chosen as

$$
u(t)=Y \widetilde{P}^{-1} x(t) .
$$

Proof. Assume that $u(t)=K x(t) ; B_{u} u(t)$ and $C_{u} u(t)$ in (1) are replaced by $B_{u} K x(t)$ and $C_{u} K x(t)$, respectively. Define $A_{k} \doteq$ 
$A+B_{u} K$ and $C_{k} \doteq C+C_{u} K$. Taking this into account, the condition in Theorem 2 is replaced by

$$
\left[\begin{array}{ccccccccc}
\Phi_{11}^{\prime} & \Phi_{12} & \Phi_{13} & \Phi_{14} & \Phi_{15} & \Phi_{16} & A_{k}^{T} H & 0 & C_{k}^{T} \\
* & \Phi_{22} & \Phi_{23} & \Phi_{24} & \Phi_{25} & \Phi_{26} & 0 & 0 & 0 \\
* & * & \Phi_{33} & \Phi_{34} & \Phi_{35} & \Phi_{36} & A_{d}^{T} H & 0 & 0 \\
* & * & * & \Phi_{44} & \Phi_{45} & \Phi_{46} & 0 & 0 & 0 \\
* & * & * & * & \Phi_{55} & \Phi_{56} & 0 & 0 & 0 \\
* & * & * & * & * & \Phi_{66} & C_{d}^{T} H & 0 & 0 \\
* & * & * & * & * & * & -H & 0 & 0 \\
* & * & * & * & * & * & * & -\gamma^{2} I & D^{T} \\
* & * & * & * & * & * & * & * & -I
\end{array}\right]<0,
$$

where

$$
\Phi_{11}^{\prime}=P A_{k}+A_{k}^{T} P+Q_{1}+N_{1}+N_{1}^{T}+h X_{11}
$$

Pre- and postmultiplying (31) by diag $\left\{P^{-T}, P^{-T}, P^{-T}\right.$, $\left.P^{-T}, P^{-T}, P^{-T}, P^{-T}, I, I\right\}$ and $\operatorname{diag}\left\{P^{-1}, P^{-1}, P^{-1}, P^{-1}, P^{-1}\right.$, $\left.P^{-1}, P^{-1}, I, I\right\}$, respectively, and introducing an additional constraint $H=\lambda P$, then one can obtain an equivalent expression of (31) as follows:

$$
\left[\begin{array}{cccccc}
\Phi_{11}^{\prime} & \bar{\Phi}_{12} & \bar{\Phi}_{13} & \bar{\Phi}_{14} & \bar{\Phi}_{15} & \bar{\Phi}_{16} \\
* & \bar{\Phi}_{22} & \bar{\Phi}_{23} & \bar{\Phi}_{24} & \bar{\Phi}_{25} & \bar{\Phi}_{26} \\
* & * & \bar{\Phi}_{33} & \bar{\Phi}_{34} & \bar{\Phi}_{35} & \bar{\Phi}_{36} \\
* & * & * & \bar{\Phi}_{44} & \bar{\Phi}_{45} & \bar{\Phi}_{46} \\
* & * & * & * & \bar{\Phi}_{55} & \bar{\Phi}_{56} \\
* & * & * & * & * & \bar{\Phi}_{66} \\
* & * & * & * & * & * \\
* & * & * & * & * & * \\
* & * & * & * & * & *
\end{array}\right.
$$

$\left.\begin{array}{ccc}\lambda P^{-T} B^{T} & & P^{-T} C^{T} \\ +\lambda P^{-T} K^{T} B_{u}^{T} & 0 & +P^{-T} K^{T} C_{u}^{T} \\ 0 & 0 & 0 \\ \lambda P^{-T} A_{d}^{T} & 0 & 0 \\ 0 & 0 & 0 \\ 0 & 0 & 0 \\ \lambda P^{-T} C_{d}^{T} & 0 & 0 \\ -\lambda P^{-T} & 0 & 0 \\ * & -\gamma^{2} I & D^{T} \\ * & * & -I\end{array}\right]<0$,

where

$$
\begin{gathered}
\bar{\Phi}_{11}^{\prime}=B_{u} K P^{-1}+P^{-T} K^{T} B_{u}^{T}+P^{-T} B^{T}+B P^{-1} \\
+\bar{Q}_{1}+\bar{N}_{1}+\bar{N}_{1}^{T}+h \bar{X}_{11}, \\
\bar{\Phi}_{12}=\bar{N}_{2}^{T}+h \bar{X}_{12}, \\
\bar{\Phi}_{13}=A_{d} P^{-1}-\bar{N}_{1}+\bar{N}_{3}^{T}+h \bar{X}_{13}, \\
\bar{\Phi}_{14}=\bar{N}_{4}^{T}+h \bar{X}_{14}, \quad \bar{\Phi}_{15}=\bar{N}_{5}^{T}+h \bar{X}_{15}, \\
\bar{\Phi}_{16}=C_{d} P^{-1}+\bar{N}_{6}^{T}+h \bar{X}_{16}, \\
\bar{\Phi}_{22}=-\left(1-\frac{\mu}{2}\right) \bar{Q}_{1}+\bar{Q}_{2}+h \bar{X}_{22}, \\
\bar{\Phi}_{23}=-\bar{N}_{2}+h \bar{X}_{23}, \\
\bar{\Phi}_{25}=h \bar{X}_{25}, \quad \bar{\Phi}_{24}=h \bar{\Phi}_{24}, h \bar{X}_{26}, \\
\bar{\Phi}_{45}=h \bar{X}_{45}, \quad \bar{\Phi}_{46}=h \bar{X}_{46}, \\
\bar{\Phi}_{33}=-(1-\mu) \bar{Q}_{2}+\bar{Q}_{3}-\bar{N}_{3}-\bar{N}_{3}^{T}+h \bar{X}_{33}, \\
-\bar{N}_{4}+h \bar{X}_{34}, \quad \bar{\Phi}_{35}=-\bar{N}_{5}+h \bar{X}_{35}, \\
\bar{\Phi}_{36}=-\bar{N}_{6}+h \bar{X}_{36}, \\
\bar{\Phi}_{34},
\end{gathered}
$$

$$
\begin{aligned}
& \bar{\Phi}_{55}=-\bar{Q}_{4}+h \bar{X}_{55}, \quad \bar{\Phi}_{56}=h \bar{X}_{56} \text {, } \\
& \bar{\Phi}_{66}=-\bar{R}+h \bar{X}_{66} \text {, } \\
& \bar{Q}_{i}=P^{-T} Q_{i} P^{-1} \quad(i=1, \ldots, 4), \\
& \bar{N}_{i}=P^{-T} N_{i} P^{-1} \quad(i=1, \ldots, 6), \\
& \bar{R}=P^{-T} R P^{-1}, \\
& \bar{X}_{i j}=P^{-T} X_{i j} P^{-1} \quad(i=1, \ldots, 6, j=1, \ldots, 6), \\
& \bar{Z}=P^{-T} Z P^{-1} \text {. } \\
& \widetilde{P}=P^{-T}, \quad Y=K \widetilde{P}, \quad \widetilde{Q}_{i}=\bar{Q}_{i} \quad(i=1, \ldots, 4), \\
& \widetilde{N}_{i}=\bar{N}_{i} \quad(i=1, \ldots, 6), \\
& \widetilde{X}_{i j}=\bar{X}_{i j} \quad(i=1, \ldots, 6, j=1, \ldots, 6), \\
& \widetilde{R}=\bar{R}, \quad \widetilde{Z}=\bar{Z},
\end{aligned}
$$

thus we can obtain (27).

The proof is thus completed.

Remark 6. It should be pointed out that Theorem 5 gives a sufficient condition for the existence of a sate feedback 
compensation controller with the $H_{\infty}$ performance bound $\gamma$ in the form of (3) for system (1), which guarantees the closedloop system to be asymptotically stable.

Remark 7. Since conditions (27) and (28) in Theorem 5 are in the LMI forms, for a given scalar $\gamma>0$, the solutions can be easily obtained using LMI Toolbox. The problem is then how to find the optimal values of $\gamma$. A feasible optimizing approach is given in Corollary 8 as follows, which can be completed using the Matlab command mincx.

Corollary 8. A suboptimal $H_{\infty}$ controller in the form of (3) for the neutral time-delay system (1) can be found by solving the following optimization problem:

$$
\begin{aligned}
& \min \gamma \\
& \text { s.t. } \quad(27),(28) .
\end{aligned}
$$

\section{Numerical Examples and Discussions}

This section presents some examples to illustrate the effectiveness of the methods described above.

Example 9. Consider the neutral system

$$
\begin{gathered}
\dot{x}(t)-C_{d} \dot{x}(t-\tau)=A x(t)+A_{d} x(t-d(t))+B w(t), \\
z(t)=C x(t)+D w(t)
\end{gathered}
$$

with

$$
\begin{array}{cc}
A=\left[\begin{array}{cc}
-1.7 & 0 \\
0 & -0.9
\end{array}\right], & B=\left[\begin{array}{c}
0.5 \\
1
\end{array}\right], \\
A_{d}=\left[\begin{array}{cc}
-1 & 0 \\
-1 & -1
\end{array}\right], & C_{d}=\left[\begin{array}{cc}
0.1 & 0 \\
0 & 0.1
\end{array}\right], \\
C=\left[\begin{array}{ll}
1 & 0
\end{array}\right], & D=0.1 .
\end{array}
$$

By Theorem 2, the $H_{\infty}$ index $\gamma$ is listed in Table 1 for various values of $\mu$ and $h$. It is clear that our results presented in this paper are feasible.

Example 10. Consider the neutral system

$$
\begin{gathered}
\dot{x}(t)-C_{d} \dot{x}(t-\tau)=A x(t)+A_{d} x(t-d(t)) \\
+B w(t)+B_{u} u(t), \\
z(t)=C x(t)+C_{u} u(t)+D w(t),
\end{gathered}
$$

with

$$
\begin{gathered}
A=\left[\begin{array}{cc}
-1 & -0.2 \\
0 & -1
\end{array}\right], \quad A_{d}=\left[\begin{array}{cc}
1 & 0 \\
0 & 1.2
\end{array}\right], \\
C_{d}=\left[\begin{array}{cc}
0.2 & 0 \\
0 & 0.1
\end{array}\right], \quad B_{u}=\left[\begin{array}{l}
1 \\
1
\end{array}\right], \quad B=\left[\begin{array}{l}
1 \\
1
\end{array}\right], \\
C=\left[\begin{array}{ll}
0 & 0.5
\end{array}\right], \quad C_{u}=0.5, \quad D=0.2 .
\end{gathered}
$$

TABLE 1: $H_{\infty}$ index $\gamma$ for various values of $\mu$ and $h$.

\begin{tabular}{lcccc}
\hline$h$ & 0 & 0.5 & 1 & $1.97 / 1.57 / 1.31$ \\
\hline$\mu=0$ & 0.2859 & 0.3205 & 0.4678 & 0.6693 \\
$\mu=0.5$ & 0.2859 & 0.3205 & 0.5158 & 0.7722 \\
$\mu=0.9$ & 0.2859 & 0.3205 & 0.6010 & 0.9340 \\
\hline
\end{tabular}

For $\mu=0.5, h=0.8, x(t)=[1-1]^{T}$, a minimum of $\gamma=$ 0.8 with a corresponding gain $K=[0.9646-12.1649]$ was obtained with Theorem 5, which implies that the proposed method is effective and feasible.

\section{Conclusions}

In this contribution, the $H_{\infty}$ performance for linear neutral system with time-varying delay is discussed and a new bounded real lemma is presented by introducing a novel Lyapunov-Krasovskii functional. Based on the BRL, an approach to design memoryless state-feedback $H_{\infty}$ controller using LMI technique for linear neutral system with timevarying delay is proposed, which can be solved readily by using existing LMI optimization techniques. The numerical example simulation results demonstrate that the method is feasible and effective. Therefore, how to further reduce the conservatism constitutes is an important problem for future investigation.

\section{Acknowledgments}

The work is supported by Development Program for Outstanding Young Teachers in Harbin Institute of Technology (HITQNJS.2009.007), National Natural Science Foundation of China (NSFC 61273094) and the Project for Distinguished Young Scholars of the Basic Research Plan in Shenzhen City under Contract No. JCJ201110001. The authors are very thankful to the reviewers for their valuable suggestions and comments.

\section{References}

[1] M. T. Alrifai, M. Zribi, M. Rayan, and M. S. Mahmoud, "On the control of time delay power systems," International Journal of Innovative Computing, Information and Control, vol. 9, no. 2, pp. 769-792, 2013.

[2] J. Zhang, P. Shi, and J. Qiu, "Robust stability criteria for uncertain neutral system with time delay and nonlinear uncertainties," Chaos, Solitons \& Fractals, vol. 38, no. 1, pp. 160-167, 2008.

[3] D. Nešić and A. R. Teel, "Input-to-state stability of networked control systems," Automatica, vol. 40, no. 12, pp. 2121-2128, 2004.

[4] R. Yang, P. Shi, G.-P. Liu, and H. Gao, "Network-based feedback control for systems with mixed delays based on quantization and dropout compensation," Automatica, vol. 47, no. 12, pp. 2805-2809, 2011.

[5] L. Wu and W. X. Zheng, "Passivity-based sliding mode control of uncertain singular time-delay systems," Automatica, vol. 45, no. 9, pp. 2120-2127, 2009.

[6] Z. Xiang, Y.-N. Sun, and M. S. Mahmoud, "Robust finite-time $H_{\infty}$ control for a class of uncertain switched neutral systems," 
Communications in Nonlinear Science and Numerical Simulation, vol. 17, no. 4, pp. 1766-1778, 2012.

[7] L. Wu and Z. Wang, "Guaranteed cost control of switched systems with neutral delay via dynamic output feedback," International Journal of Systems Science, vol. 40, no. 7, pp. 717-728, 2009.

[8] A. G. Wu and G. R. Duan, "On delay-independent stability criteria for linear time-delay systems," International Journal of Automation and Computing, vol. 4, no. 1, pp. 95-100, 2007.

[9] P.-A. Bliman, "LMI characterization of the strong delay-independent stability of linear delay systems via quadratic Lyapunov-Krasovskii functionals," Systems \& Control Letters, vol. 43, no. 4, pp. 263-274, 2001.

[10] L. Wu and W. X. Zheng, "Weighted $H_{\infty}$ model reduction for linear switched systems with time-varying delay," Automatica, vol. 45, no. 1, pp. 186-193, 2009.

[11] M. S. Mahmoud, "Robust $H_{\infty}$ control of linear neutral systems," Automatica, vol. 36, no. 5, pp. 757-764, 2000.

[12] E. Fridman and U. Shaked, "Delay-dependent stability and $H_{\infty}$ control: constant and time-varying delays," International Journal of Control, vol. 76, no. 1, pp. 48-60, 2003.

[13] J.-H. Park, "A new delay-dependent criterion for neutral systems with multiple delays," Journal of Computational and Applied Mathematics, vol. 136, no. 1-2, pp. 177-184, 2001.

[14] L. Wu, X. Su, and P. Shi, "Sliding mode control with bounded $L_{2}$ gain performance of Markovian jump singular time-delay systems," Automatica, vol. 48, no. 8, pp. 1929-1933, 2012.

[15] R. Yang, Z. Zhang, and P. Shi, "Exponential stability on stochastic neural networks with discrete interval and distributed delays," IEEE Transactions on Neural Networks, vol. 21, no. 1, pp. 169-175, 2010.

[16] A. Wu, H. Dong, and G. Duan, "Improved robust $H$-infinity estimation for uncertain continuous-time systems," Journal of Systems Science \& Complexity, vol. 20, no. 3, pp. 362-369, 2007.

[17] S. Xu, J. Lam, and Y. Zou, "New results on delay-dependent robust $H_{\infty}$ control for systems with time-varying delays," Automatica, vol. 42, no. 2, pp. 343-348, 2006.

[18] A. Rodkina and M. Basin, "On delay-dependent stability for a class of nonlinear stochastic delay-differential equations," Mathematics of Control, Signals, and Systems, vol. 18, no. 2, pp. 187197, 2006.

[19] F. Qiu, B. Cui, and Y. Ji, "Further results on robust stability of neutral system with mixed time-varying delays and nonlinear perturbations," Nonlinear Analysis, vol. 11, no. 2, pp. 895-906, 2010.

[20] M. Wu, Y. He, and J.-H. She, "New delay-dependent stability criteria and stabilizing method for neutral systems," IEEE Transactions on Automatic Control, vol. 49, no. 12, pp. 22662271, 2004.

[21] L. Wu, X. Su, P. Shi, and J. Qiu, "A new approach to stability analysis and stabilization of discrete-time T-S fuzzy timevarying delay systems," IEEE Transactions on Systems, Man, and Cybernetics B, vol. 41, no. 1, pp. 273-286, 2011.

[22] F. Gouaisbaut and D. Peaucelle, "Delay-dependent stability analysis of linear time delay systems," in Proceedings of the 6th IFAC Workshop on Time Delay Systems (TDS '06), pp. 1-12, L'Aquila, Italy, July 2006.

[23] Y. B. Zhang and J. M. Li, "Improved stability criteria for neutral delay systems," Applied Mathematics, vol. 26, no. 3, pp. 355-362, 2011.

[24] Y. S. Lee, Y. S. Moon, W. H. Kwon, and P. G. Park, "Delaydependent robust $H_{\infty}$ control for uncertain systems with a state-delay," Automatica, vol. 40, no. 1, pp. 65-72, 2004.
[25] H. W. Xia, G. C. Ma, C. H. Wang, and C. Ma, "Robust $H_{\infty}$ filtering for a class of uncertain networked control systems with limited communication," Control and Decision, vol. 23, no. 8, pp. 888-893, 2008.

[26] L. Hu, H. Gao, and W. X. Zheng, "Novel stability of cellular neural networks with interval time-varying delay," Neural Networks, vol. 21, no. 10, pp. 1458-1463, 2008.

[27] Q.-L. Han, "Improved stability criteria and controller design for linear neutral systems," Automatica, vol. 45, no. 8, pp. 1948-1952, 2009.

[28] Y. He, Q.-G. Wang, L. Xie, and C. Lin, "Further improvement of free-weighting matrices technique for systems with timevarying delay," IEEE Transactions on Automatic Control, vol. 52, no. 2, pp. 293-299, 2007.

[29] L. Wu, X. Su, P. Shi, and J. Qiu, "Model approximation for discrete-time state-delay systems in the TS fuzzy framework," IEEE Transactions on Fuzzy Systems, vol. 19, no. 2, pp. 366-378, 2011.

[30] A. G. Wu, J. Dong, and G. R. Duan, "Robust H-infinity estimation for linear time-delay systems: an improved lmi approach," International Journal of Control, Automation and Systems, vol. 7, no. 4, pp. 668-673, 2009.

[31] S. Mou, H. Gao, J. Lam, and W. Qiang, "A new criterion of delaydependent asymptotic stability for Hopfield neural networks with time delay," IEEE Transactions on Neural Networks, vol. 19, no. 3, pp. 532-535, 2008.

[32] O. Kwon, M. Park, J. Park, S. Lee, and E. Cha, "New delay-partitioning approaches to stability criteria for uncertain neutral systems with time-varying delays," Journal of the Franklin Institute, vol. 349, no. 9, pp. 2799-2823, 2012.

[33] W. Chen, Q. Ma, G. Miao, and Y. Zhang, "Stability analysis of stochastic neural networks with Markovian jump parameters using delay-partitioning approach," Neurocomputing, vol. 103, pp. 22-28, 2013. 


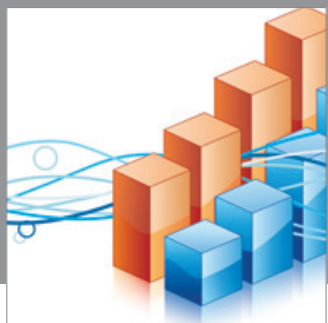

Advances in

Operations Research

mansans

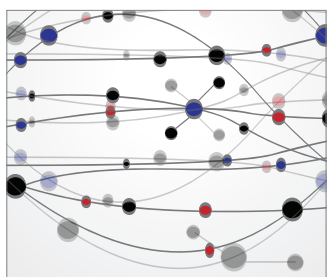

The Scientific World Journal
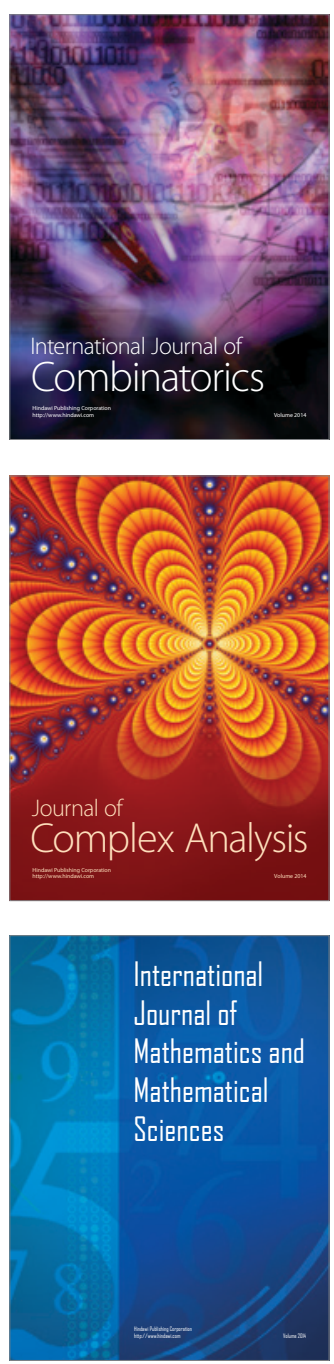
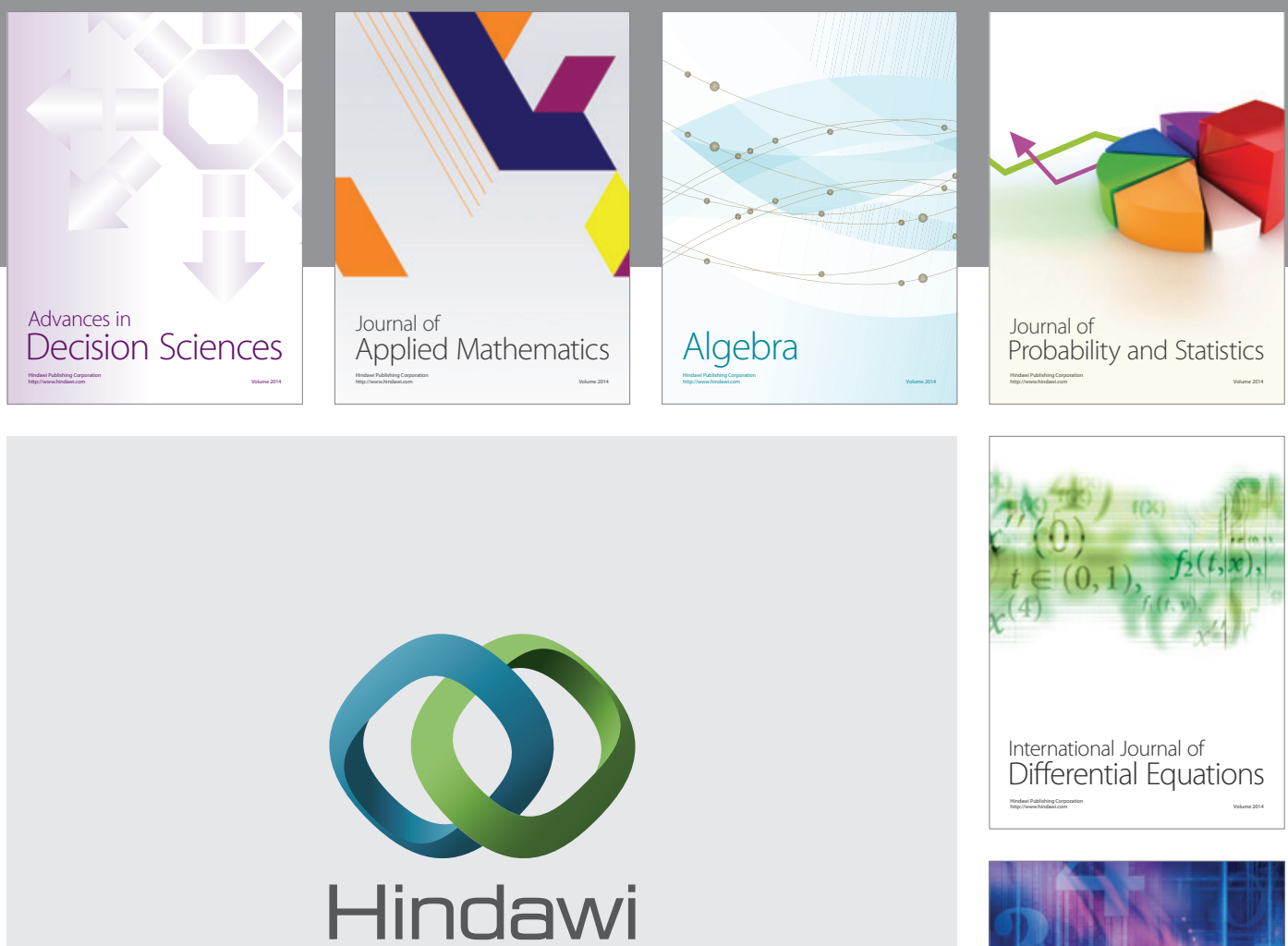

Submit your manuscripts at http://www.hindawi.com
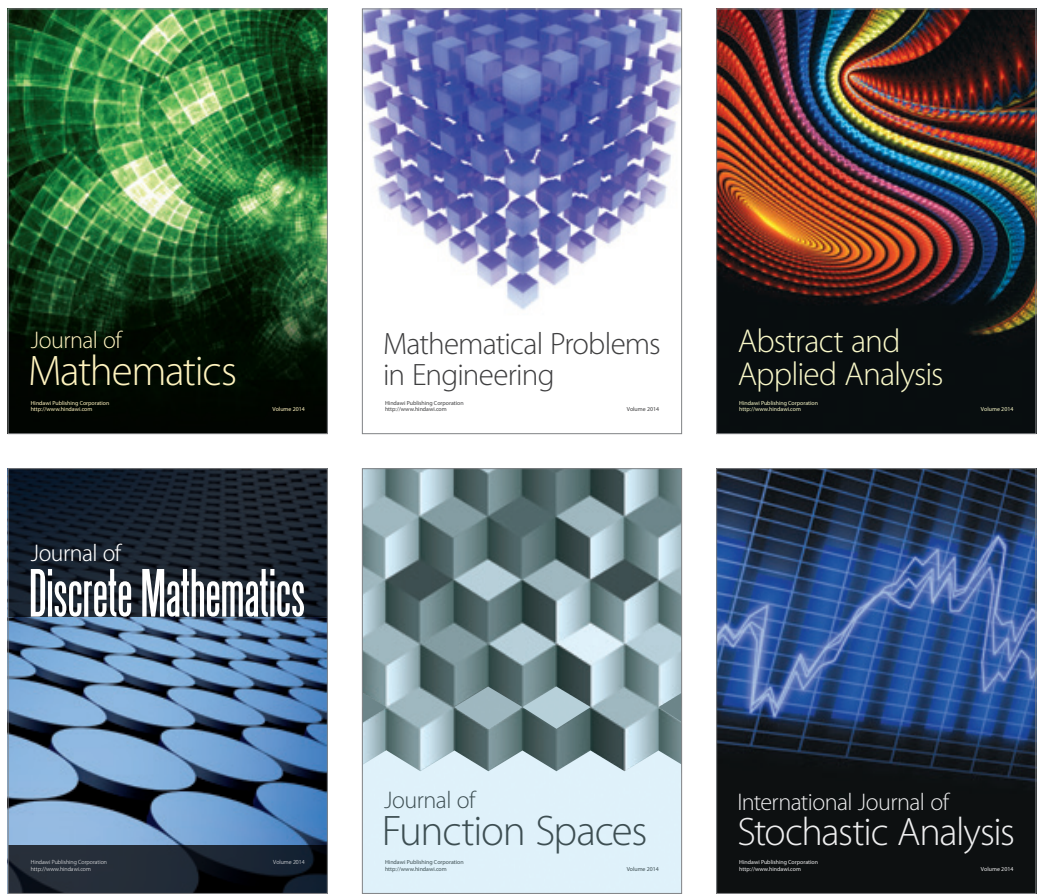

Journal of

Function Spaces

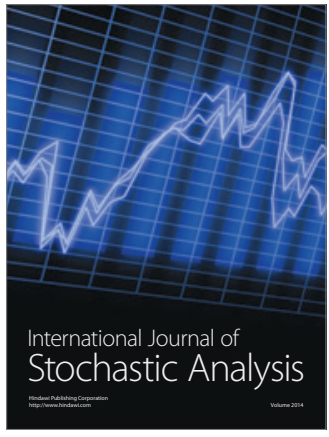

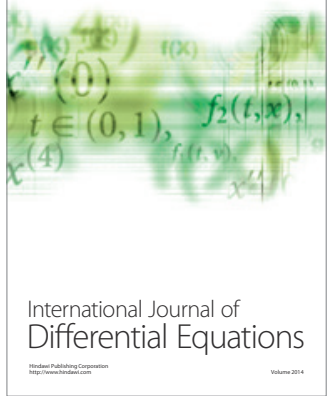
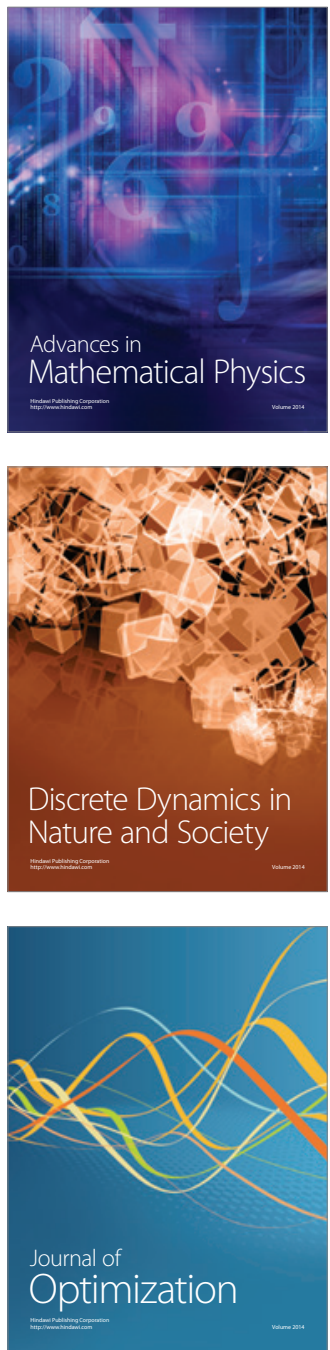